\title{
América
}

Cahiers du CRICCAL

43 | 2013

Le Crime (vol. 1)

II. Drôles de genres, ou les poétiques de la représentation

\section{Le crime et le macabre sur scène dans La infanta Oriana (1852), comédie de magie de Manuel Fernández González}

El crimen y lo macabro escenificados en La infanta Oriana, comedia de magia de Manuel Fernández

\section{LISE JANKOVIC}

p. $79-90$

\section{Résumés}

\section{Français Español}

L'étude de la figuration du crime dans la « comédie de magie » espagnole de la fin du XIXe siècle réserve des surprises. Alors qu'on pouvait croire que dans de telles œuvres, le crime ne pouvait être que surnaturel, nous découvrons qu'en réalité la représentation de l'assassinat dans ce théâtre magique est dénuée d'effets merveilleux. En revanche, ce qui caractérise cette " comédie de magie » est que le crime n'envisage pas la mort comme irréversible, définitive. La résurrection montre les grandes libertés dramaturgiques de ce genre populaire. Libertés dramaturgiques, mais pas idéologiques, puisque l'analyse de la figuration du crime démontre un manichéisme qui structure les pièces magiques où le criminel finit toujours par recevoir le châtiment que lui inflige le Bien triomphant.

Estudiar la figuración del crimen en la comedia de magia española del siglo XIX conlleva sorpresas. Cuando se podía imaginar que en semejantes obras el crimen no puede sino ser sobrenatural, descubrimos en realidad que la representación del asesinato en el teatro mágico decimonónico está desprovista de efectos maravillosos. En cambio, lo que sí es 
característico de la comedia de magia es que el crimen no supone una muerte irreversible y definitiva. La resurrección refleja las grandes libertades dramatúrgicas de este género popular. Libertades dramatúrgicas, pero no ideológicas, puesto que el análisis de la figuración del crimen demuestra el maniqueísmo estructurador de las obras de magia en que el criminal siempre acaba siendo castigado por el Bien triunfador.

\section{Entrées d'index}

Mots-clés : théâtre, magie, mise en scène, triomphe du bien

Palabras claves : teatro, magia, escenificación, triunfo del bien

Notes de l'auteur

Manuel Fernández González (1821-1888) : romancier feuilletoniste, andalou.

\section{Texte intégral}

Les contes de fées ruissellent de sang. Les impulsions meurtrières, la cruauté, les tortures sont leur climat habituel. (Henri-René Lenormand ${ }^{1}$ )

Des CORPS SANS VIE TOMBENT SUR SCÈne depuis le théâtre classique; ils tombent sous les yeux du public : spectacle de la mort qui fait tressaillir. Le crime est généralement perpétré à l'encontre des héros et il est surtout réservé à la tragédie. Pensons : au double assassinat vindicatif de Federico et Casandra dans El castigo sin venganza, de Lope de Vega (1631), au meurtre de doña Mencia orchestré par don Gutierre, son mari jaloux dans El médico de su honra, de Calderón (1637), au crime passionnel commis par Orosmane dans Zaïre, de Voltaire (1632), argument repris par le dramaturge espagnol García de la Huerta dans La fe triunfante del amor y cetro o Xaira (1784) ou encore au meurtre de don Gonzalo et de don Luis par le Don Juan Tenorio de José Zorrilla (1844).

Dans la tradition théâtrale, l'on observe ainsi le passage du crime hors scène (raconté sur scène par hypotypose) au crime sur scène. La règle aristotélicienne de bienséance a, en effet, longtemps prévalu :
La frayeur et la pitié peuvent assurément naître du spectacle, mais elles peuvent naître aussi du système des faits lui-même : c'est là le procédé qui tient le premier rang et révèle le meilleur poète. Il faut en effet qu'indépendamment du spectacle l'histoire soit ainsi constituée qu'en apprenant les faits qui se produisent on frissonne et on soit pris de pitié devant ce qui se passe : c'est ce qu'on ressentirait en écoutant l'histoire d'Edipe. Produire cet effet par les moyens du spectacle ne relève guère de l'art : c'est affaire de mise en scène. Ceux qui, par les moyens du spectacle, produisent non l'effrayant, mais seulement le monstrueux, n'ont rien à voir avec la tragédie ; car [...] le plaisir que doit produire le poète vient de la pitié et de la frayeur éveillées par l'activité représentative. (Aristote, La Poétique, chapitre 14)

3 Du point de vue dramaturgique, les crimes supposent tous une victime, un agresseur, adversaire ou ennemi, un affrontement, des armes, du sang, des larmes souvent, c'est-à-dire tout un système de personnages s'inscrivant dans un réseau relationnel sujets/opposants, selon une dualité très fortement antagonique.

Les mobiles du crime au théâtre sont variés : coup de poignard d'un traitre, crime passionnel (suite à un quiproquo dans Zä̈re), meurtre prémédité, réparation de l'honneur par un duel meurtrier, assassinat politique, homicide 
vindicatif. Et les armes du crime sont généralement blanches (poignard, glaive, dague, épée...).

\begin{abstract}
La catastrophe criminelle a pour objet un acte extrêmement grave et noir, majoritairement irrémédiable, dont le moment paroxystique tient en un crime, c'est-à-dire un manquement fondamental à la morale et à la loi. Si certains actes criminels n'entraînent pas la mort comme le viol ou l'inceste, la plupart d'entre eux lui sont associés, tels le sacrifice, le suicide souvent sacrificiel et le meurtre. Pour créer un maximum de terreur et de pitié, le crime doit être commis entre personnages proches, qu'ils soient amis, amants, ou parents. (Bouissou, 2011: 53)
\end{abstract}

Une idée nous intéresse ici, au regard de la comédie de magie, c'est celle de crime comme climax de la tension dramatique ; nous observerons comment cette norme est détournée.

Voyons donc pourquoi le sang est versé dans la comédie de magie et comment il est figuré. S’il a lieu dans une comédie de magie, doit-on s'attendre à ce que le crime soit commis de façon surnaturelle et ténébreuse ? Non, mais en revanche la mort n'est pas sans retour, car, comme dans le conte, la résurrection est possible pour la victime. C'est cette possibilité du retour à la vie qui fait apparaître d'emblée les enjeux propres à la comédie de magie concernant la figuration de la catastrophe mortifère.

$7 \quad$ Voici un résumé de l'intrigue de la pièce de Manuel Fernández González.

8 La pièce met en scène la vengeance de l'infante Oriana et du roi Arthur, victimes d'un enchantement de Merlin (elle est condamnée à être une vieille sorcière jusqu'au jour où un écuyer acceptera de l'épouser ; Arthur, lui, a été envoyé au royaume des morts). Merlin a tué le comte de Castrojeriz, se fait passer pour son frère et veut épouser sa nièce doña Sol. Mais don Alvar, accompagné de son écuyer Chorlito, est épris de la jeune femme et l'infante se fait son alliée. Don Alvar est tué par des veneurs engagés pour surveiller le château, mais il ressuscite grâce à une potion de l'infante. Merlin et son complice don Gaiferos sont faits prisonniers par Oriana ; doña Sol échappe ainsi de justesse au mariage forcé que Merlin voulait célébrer à la hâte. L'enchanteur s'enfuit de la tour où il est enfermé grâce à la Dame Blanche et à des génies, puis il demande de l'aide à Lucifer. À son tour, il emprisonne les jeunes amants qui réussissent à s'échapper grâce à un parchemin récupéré chez la Célestine lors du sabbat des sorcières. Don Alvar apprend que le comte de Castrojeriz était son père et il est fait chevalier par le fantôme du roi Arthur. A la suite d'un duel entre Merlin et don Alvar, le chevalier fait tout avouer à l'enchanteur qui confesse la vérité et s'engouffre en Enfer.

\title{
1. Un cadre propice à la figuration du crime
}

La mort est omniprésente sur scène, dans le décor comme dans l'action, tout au long de la comédie, enveloppant les spectateurs dans une atmosphère macabre. Au lever de rideau, le lieu scénique représente « la selva de la fantasma ». La forêt est un topos du merveilleux, omniprésente dans les contes, légendes, féeries théâtrales et récits fantastiques : on s'y perd, on y rencontre des créatures magiques ; la forêt est un lieu de mise à l'épreuve, un espace où la frontière devient floue entre le monde réel et surnaturel, car elle abrite des forces mystérieuses. D'autant plus que l'espace sylvestre est ici en relation avec le monde 
des ombres, puisque la pièce débute dans un lieu hanté et que la didascalie initiale situe l'action à la tombée du jour, plongeant la scène dans une atmosphère crépusculaire propice au crime.

Plus avant dans la pièce, d'autres décors prononcent le trait lugubre du lieu scénique, avec notamment un panthéon souterrain (acte III, 10e tableau) "Panthéon souterrain : au fond, une galerie obscure ; à droite de l'acteur, une sépulture et une autre à gauche; toutes deux au deuxième plan. Derrière celle de droite, un autel illuminé par une lampe et au-dessus, la statue de l'ange de la mort. $»^{2}$ (figure 1) Une esthétique lugubre très typiquement romantique. Et l'Enfer est représenté à l'avant-dernière scène (12e tableau) : "Vestibule en Enfer ; au fond, le trône de Satan »3.

11 Ces décors sont complétés par de sinistres objets scéniques qui parsèment la scène : crochets, tenailles, ossements, têtes de mort et squelettes, sans oublier un parchemin aux lettres ensanglantées. Le crime macule et le sang est sa connotation scénique visuelle la plus évidente et immédiate.

Parmi le personnel dramatique, on compte " un horrible génie »,4 des diables bourreaux et autres créatures démoniaques, 5 Satan lui-même, accompagné de " génies infernaux qui improvisent une danse furieuse " ${ }^{6}$ des sorciers et sorcières, lors du sabat qui clôt le deuxième acte à minuit et l'ange de la mort, décrit comme un « ange terrible et sombre / qui par ordre de Dieu, / [est] le gardien des tombes ${ }^{7}$.

Certains de ces personnages font partie du personnel dramatique féerique habituel (les génies, les diables et les ensorceleurs), mais La infanta Oriana met clairement l'accent sur le macabre avec la torture, le culte nocturne du diable et l'ange de l'au-delà. Il est très caractéristique que la comédie de magie puise dans un imaginaire composite, où se meuvent toutes sortes de figures légendaires, mais aussi bibliques et mythologiques. L’important est de convoquer sur scène l'étrange et le merveilleux en représentant des êtres instantanément - et visuellement, plastiquement - identifiables comme surnaturels (figures 2, 3 et 4). La tendance est à la surenchère : plus on en rajoute, plus la comédie est magique. Les pièces féeriques n'ont pas peur du pot-pourri pourvu que le spectateur en ait plein les yeux. Tout se passe comme si l'efficacité du spectacle reposait sur ces constellations de références imaginaires.

14 Les personnages semblent cernés par la mort. Nombre de revenants peuplent la scène et s'entretiennent avec les vivants : l'ombre du roi Arthur qui apparaît au début et à la fin de la pièce, celle du comte Tello de Castrojeriz (qui a été assassiné par Merlin), au troisième acte, et celle du marquis de Villena, aux deuxième et troisième actes. Ces apparitions fantomatiques se font toutes selon le même schéma de représentation : invocation - ouverture du tombeau - apparition disparition.

\footnotetext{
- Oriana : « Ombre qui repose ici / sous ce marbre froid, / réveille-toi à ma voix / qui depuis le monde t'appelle, / et éclaire-toi de cette flamme / triste et sombre lueur. » (L'infante dépose les feuillages devant le monument. Ils s'enflamment et déploient une lumière très vive et violente. La scène, qui au fur et à mesure du monologue de l'infante s'est obscurcie au maximum, s'illumine d'un éclair ; une détonation retentit, le monument de pierre s'ouvre et le roi Arthur de Bretagne apparaît [...]. Le roi Arthur disparaît et le monument se referme. $)^{8}$
} performatif, car la parole enchantée, le plus souvent invocatoire et toutepuissante, entraîne une action presque simultanée. 
Merlin, qui est lié à la légende arthurienne depuis le xire siècle, a toujours eu un aspect inquiétant. Dans la pièce, le dramaturge en fait une figure malfaisante et criminelle qui menace tous les personnages : il a tué le père et la mère de don Alvar et trompe son monde par un travestissement, comme on l'apprend dans la lettre du père qui révèle ses crimes : "Mon fils don Alvar, quand tu liras ce parchemin, le malheur sera tombé sur toi, et tu seras enfermé dans la même tour où sera mort ton père. Venge-moi! Un infâme assassin a tué ta mère, et j'entends les pas du bourreau qui s'approche ${ }^{9}$. Classique anagnorèse, mais fort morbide. Et à la fin de la pièce, l'enchanteur sans pitié veut tuer doña Sol (de dépit) : « Elle mourra, / La tuer !... Idée terrible ! / La voir jouir... rire / dans les bras d'un autre homme ! / Qu'elle meure mille fois avant cela ! ${ }^{10}$. Il a le rôle du Méchant et incarne parfaitement celui d'opposant.

17 Le décor est donc planté : la scène, les éclairages, le personnel dramatique, tout concourt à figurer la mort et le macabre et participe à première vue à la tension dramatique.

\section{Détournement féérique et comique de la représentation du crime}

L'histoire du théâtre a eu ses heures sanglantes avec un théâtre baroque macabre (de la fin du xvie jusqu'aux années 1630-1640), dont les pièces emblématiques sont La tragédie du vengeur, de Thomas Middleton (1606) et Hypermnestre, de Gervais (1716). Un théâtre où règne la « joie maligne » qui conduit au " meurtre voluptueux » (Cavaillé, 2005 : 89). Dans ces pièces, tous les effets les plus atroces et sadiques étaient permis, avec un goût prononcé pour l'horrible et l'insoutenable, ce que Claude-Gilbert Dubois nomme le « baroquisme théâtral » (Dubois, 2000 : 140). Or la comédie de magie se définit aussi par son caractère spectaculaire, car elle se nourrit d'effets scéniques et n'existe que pour le spectateur. Spectacle par excellence, elle est prodigue en décors, accessoires et trucs, avec une surenchère dans le faste et la complexité de l'appareil scénique. En ce sens, le théâtre féerique est baroque dans son esthétique du débord, mais, lorsqu'il figure le crime sur scène, ce théâtre s'éloigne totalement des pièces baroques macabres, car dans la comédie de magie, le crime n'implique pas une mort violente ou gore et elle n'est pas définitive, puisque l'on peut ressusciter.

Il s'opère ainsi un double détournement du macabre à la fois comique et féerique. On le voit bien dans le spectacle qui est donné de l'assassinat. Le crime a lieu sur scène (I, 2, 13 et 14) : don Alvar et doña Sol s'entretiennent de leur amour, doña Sol lui apprend que le faux don Tello veut l'épouser dans la nuit et il lui propose de s'enfuir avec lui. Les musiciens qui accompagnent don Alvar chantent. Mais les veneurs au service du faux comte surviennent et tuent don Alvar. Tout se passe à la vitesse de l'éclair, la didascalie n'est pas du tout descriptive :

(Les veneurs attaquent les musiciens.) - Les musiciens : Pitié ! Au secours ! On nous assassine ! - Gaiferos : Tuez-les, tuez-les, mes amis ! Alvar : Barbares ! Mon épée... Pauvre de moi ! Confession! (Ils se battent, don Alvar est blessé et tombe) - Sol : Mon Dieu ! Il est mort! (Elle tombe évanouie derrière les créneaux) ${ }^{11}$. 
- Oriana : Donne-moi cette potion / que tu as conquise à Fierabrás [...]

- Artus : Qui va mourir?

- Oriana : Un jeune homme / à qui Merlin arracha / père et nom [...] il se nomme Alvar12.

Une prédiction très typiquement cabalistique qui instaurait le suspens et plaçait le spectateur dans l'expectative de ce crime.

Et ce sont les réactions qui vont clairement tirer la scène vers le comique, car la traditionnelle scène de déploration perd ici tout caractère tragique. Chorlito, attristé par la mort de son maître, prononce un monologue qui porte à rire plus qu'à pleurer, car après un court instant d'accablement, la panique l'envahit :

Mort ! oui, il est rigide, très rigide, j'aimerais pleurer... j'aimerais bien... mais c'est que je ne sais pas... ce que je veux, ce que je sais indubitablement... je sais... ce que je veux c'est déguerpir d'ici vite fait bien fait 13 .

Pas le temps de se lamenter sur l'injustice du sort, plutôt se mettre à l'abri le plus vite possible ! Le crime est traité ici de façon fort légère.

Puis, c'est le retour à la vie qui va constituer le second opérateur de détournement. Le thème de la résurrection a traversé les siècles dans tous les arts. Dans la mythologie, elle est souvent poétisée à travers la métamorphose et le passage à un autre règne et, à partir de l'ère chrétienne, ce miracle a une résonance évidemment christique.

La foi en la résurrection des corps fait partie des caractères originaux du christianisme. À la différence d'autres dogmes, comme celui de l'Incarnation, la résurrection personnelle, celle du dernier jour, pour la damnation ou pour le bonheur éternel, est bien distincte de celle du Christ, prémonitoire mais unique : le Christ, premier né d'entre les morts, reste différent de chacun des ressuscités futurs. Le christianisme postule donc le maintien de la singularité personnelle après la mort comme une donnée centrale de la foi. (Lemaître, Nicole, préface in Molinié, 2007 : 7)

Dans les contes, auxquels le théâtre féerique est intimement lié, ce thème est récurrent et a été analysé par Bruno Bettelheim comme le signe d'une adolescence encore immature. La mort temporaire serait une régression, une période de gestation latente avant la pleine maturité.

De nombreux héros de contes de fées, à un moment crucial de leur développement, tombent dans un profond sommeil ou sont ramenés à la vie. Chaque réveil (ou renaissance) symbolise l'accession à un niveau supérieur de maturité et de compréhension. C'est l'une des façons qu'adopte le conte de fées pour stimuler notre désir de donner à la vie une signification plus élevée : une conscience plus profonde, une meilleure connaissance de soi et une plus grande maturité. [...] Les enfants comprendront le réveil de l'héroïne après un long sommeil de différentes façons, selon leur âge. Le tout jeune enfant y verra surtout l'éveil de sa personnalité, la mise en harmonie des tendances chaotiques qui grouillaient en lui, autrement dit l'entente harmonieuse de son ça, de son moi et de son surmoi. (Bettelheim, $1976: 314,346)$

Dans la comédie de magie, on est loin de la résurrection poétique par métamorphose (par exemple d'Atys changé en pin dans l'opéra de Lully), on est loin également de la solennelle résurrection biblique et la courte mort de don Alvar nous empêche de l'interpréter comme une période initiatique et de maturation : 


\begin{abstract}
- Oriana : Par le pouvoir du baume de Fierabrás que cette fiole contient, reviens à la vie, chevalier ensanglanté (don Alvar commence à revenir à lui.) - Chorlito : Vous devez être un diable, et non une infante ! Voyez, mon seigneur ouvre déjà les yeux. - Alvar : Où suis-je ? Que m'est-il arrivé ? Suis-je toujours en vie ? Oui, je vis. (Il se lève.) - Chorlito : Je n'oserais le jurer14.
\end{abstract}

Le retour à la vie est progressif : le chevalier revient d'abord à lui, puis il ouvre les yeux et prend la parole. La dernière phase de la résurrection de don Alvar est le moment où il se met debout. Anita Guerreau-Jalabert rappelle ce pouvoir des fées sur la mort et montre que leurs capacités guérisseuses sont souvent évoquées et symbolisées par des onguents aux vertus particulières (Guerreau-Jalabert, 1994 : 139). Pas d'éclat dans la représentation de l'événement magique : l'effet du baume est immédiat et la résurrection ressemble à un simple réveil. Il semblerait donc que ce miracle vise avant tout à prouver l'évidence de la toute-puissance de la magie, tout comme les autres prouesses surnaturelles de la pièce (transformation d'un arbre en génie, effondrement d'une tour, entre autres). Et le crime qui précède n'est en fait qu'un prétexte à la représentation d'un prodige de sorcellerie. Ainsi, au détournement comique s'ajoute bien un détournement féerique de la mort après homicide.

\title{
3. Apothéose du Bien vainqueur contre le Mal
}

Figurer le crime, puis la résurrection de la victime, c'est, dans le théâtre féerique, montrer la supériorité de la Magie Blanche sur la Magie Noire. Le crime, c'est le mal, selon un schéma manichéen que les comédies de magie partagent avec le conte. Cela permet de mieux représenter la victoire du féerique bienveillant sur les forces occultes malfaisantes. En d'autres termes : le crime et le diable contre la féerie et Dieu.

Cet affrontement de forces antagoniques est un leitmotiv assez structurel dans les pièces :

La pièce magique crée l'illusion « interne » d'avoir satisfait les besoins et résolu les problèmes des personnages, puisqu'elle remplit son rôle et châtie les méchants, de façon peu commune dans la réalité de tous les jours, pour récompenser les gentils15. (Álvarez Barrientos, 1992 : 341-349)

Une caractéristique qu'elles partagent avec l'opéra et le théâtre baroques :

L'homme heureux n'a pas d'histoire. Pour que les spectateurs vibrent au gré d'une intrigue, il faut que les héros aient des obstacles à franchir, des défis à relever, des oppositions à combattre. Il faut des gentils et surtout des méchants. Le Bien ne prend toute sa dimension émotionnelle que s'il rencontre une résistance à travers ses opposants. Les sentiments qu'il véhicule - tendresse, amour, loyauté, bravoure, franchise - ne sont jamais plus convaincants que lorsque les forces du Mal, dangereuses, maléfiques, voire mortelles, les menacent et les agressent. [...] À l'instar des auteurs dramatiques, les auteurs de théâtre lyrique ont bien compris l'enjeu de la portée du Mal et ont conféré un soin tout particulier à ses représentations surtout dans la tragédie, mais aussi dans le ballet, la pastorale et, dans une moindre mesure, la comédie. (Bouissou, 2011 : 9)

Ainsi, la seconde mort qui est offerte aux yeux du spectateur, quand, à la fin de 
la pièce, il assiste à la condamnation à mort - cette fois-ci sans retour - du maléfique enchanteur, matérialise sur scène la justice divine. Une condamnation sans appel qui convoque, elle, des effets scéniques importants, contrairement à la mise en scène de la mort et de la résurrection de don Alvar, (volontairement ?) dépouillée de tout artifice :

- Oriana : Ton heure est arrivée ; la mort te cerne les yeux. Sois enseveli.
Les portes de l'éternité te sont déjà ouvertes... Va-t'en rejoindre l'Enfer !...
Et moi je rejoins mes donjons ! - Merlin : Damnation ! (Il disparaît par la
trappe, parmi les flammes) ${ }^{16}$.

Le combat du Bien contre le Mal est très palpable dans ces répliques finales : sentence vindicative, pronoms personnels sujets en opposition, punition représentée par la descente, mouvement qui symbolise et signifie le châtiment, les flammes qui figurent la damnation - comme pour don Juan. Les forces maléfiques ne triomphent d'ailleurs jamais dans la comédie de magie et l'ordre (divin) est toujours rétabli à la fin des pièces, ce qui rappelle leur lien de parenté avec les spectacles paraliturgiques du Moyen-Âge et les comédies de saints. Un manichéisme structurel sans doute accentué pour éloigner tout soupçon d'hérésie, car le genre était surveillé de près par les autorités chargées de superviser les répertoires et a même été interdit à deux reprises en 1788 et en 1799. Grâce à ce châtiment final du sorcier - et donc du paganisme -, la comédie est marquée de l'honorable sceau de la chrétienté.

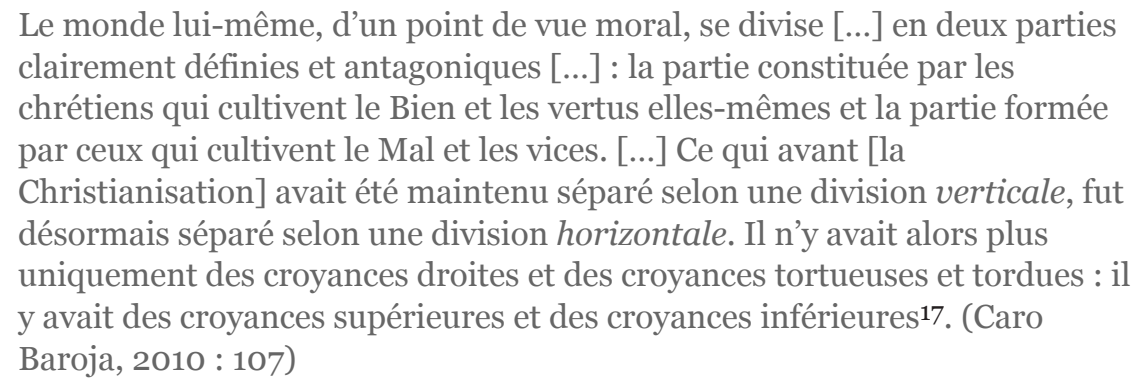

En conclusion, lorsqu'il s'agit d'un crime à l'encontre du Bien, la mort est représentée dans un contexte macabre, mais de façon sobre - pourtant, la comédie de magie est un genre dramatique dans lequel abondent les effets scéniques activés grâce à une complexe machinerie. On pourrait croire, alors, que le crime n'est qu'épiphénomène dans la pièce, mais l'on comprend, quand le mal est châtié à la fin de l'intrigue et que les trucs visuels et auditifs sont déployés, que le crime n'est pas entendu comme climax de la tension dramatique - comme c'est le cas pour le théâtre macabre baroque et la tragédie classique - mais comme un événement pouvant structurer l'action en deux tableaux antithétiques : mort du gentil don Alvar vs. mort du méchant Merlin. Au fond, ce n'est jamais le crime qui est sous les projecteurs dans la comédie de magie, mais la Magie Blanche et le Bien.

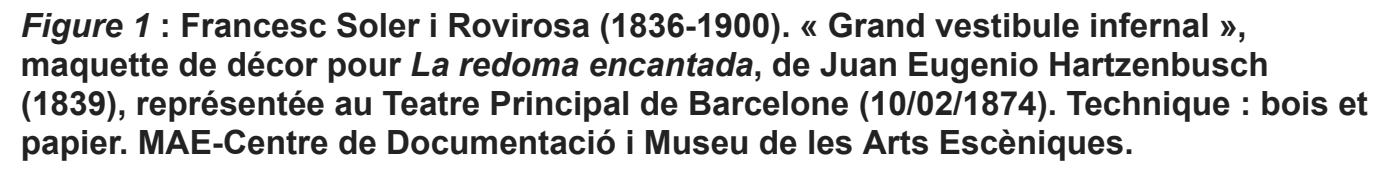




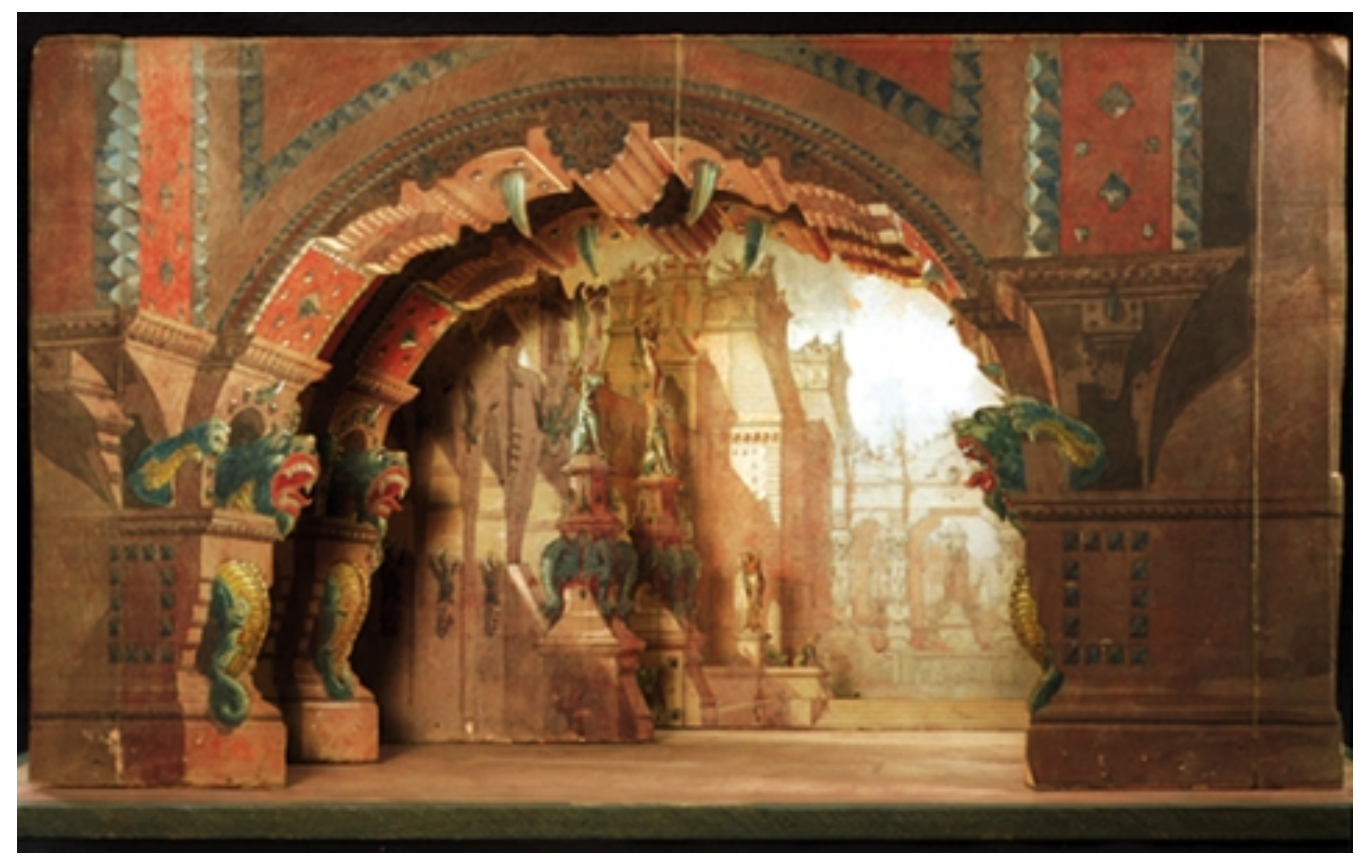

Figure 2 : Apel-les Mestres (1854-1936), maquette de costumes pour La almoneda del diablo, de Rafael María Liern (1864). Technique : aquarelle. MAE-Centre de Documentació i Museu de les Arts Escèniques.

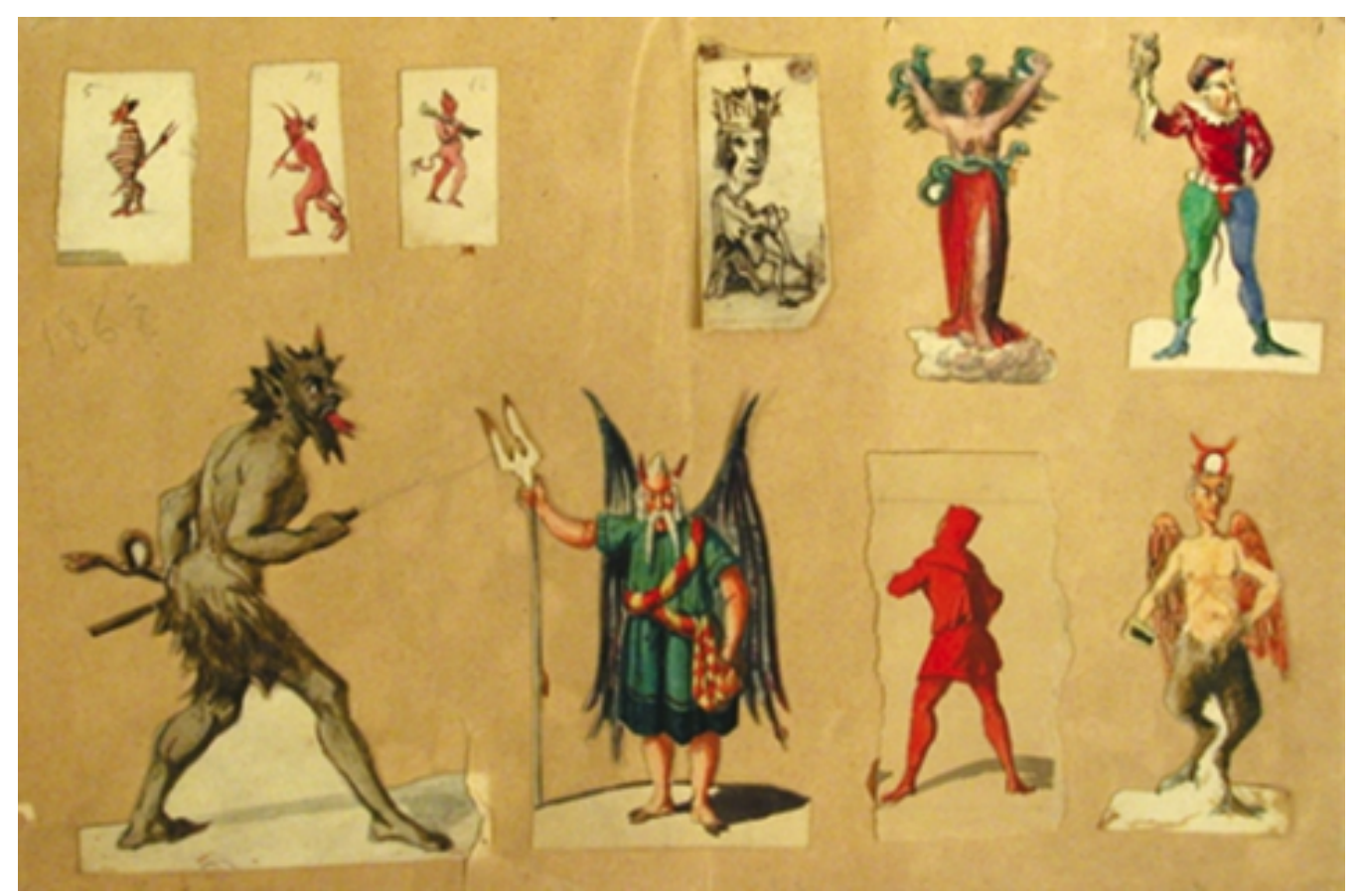

Figure 3 : Apel-les Mestres (1854-1936), maquette de costumes. MAE-Centre de Documentació i Museu de les Arts Escèniques. 


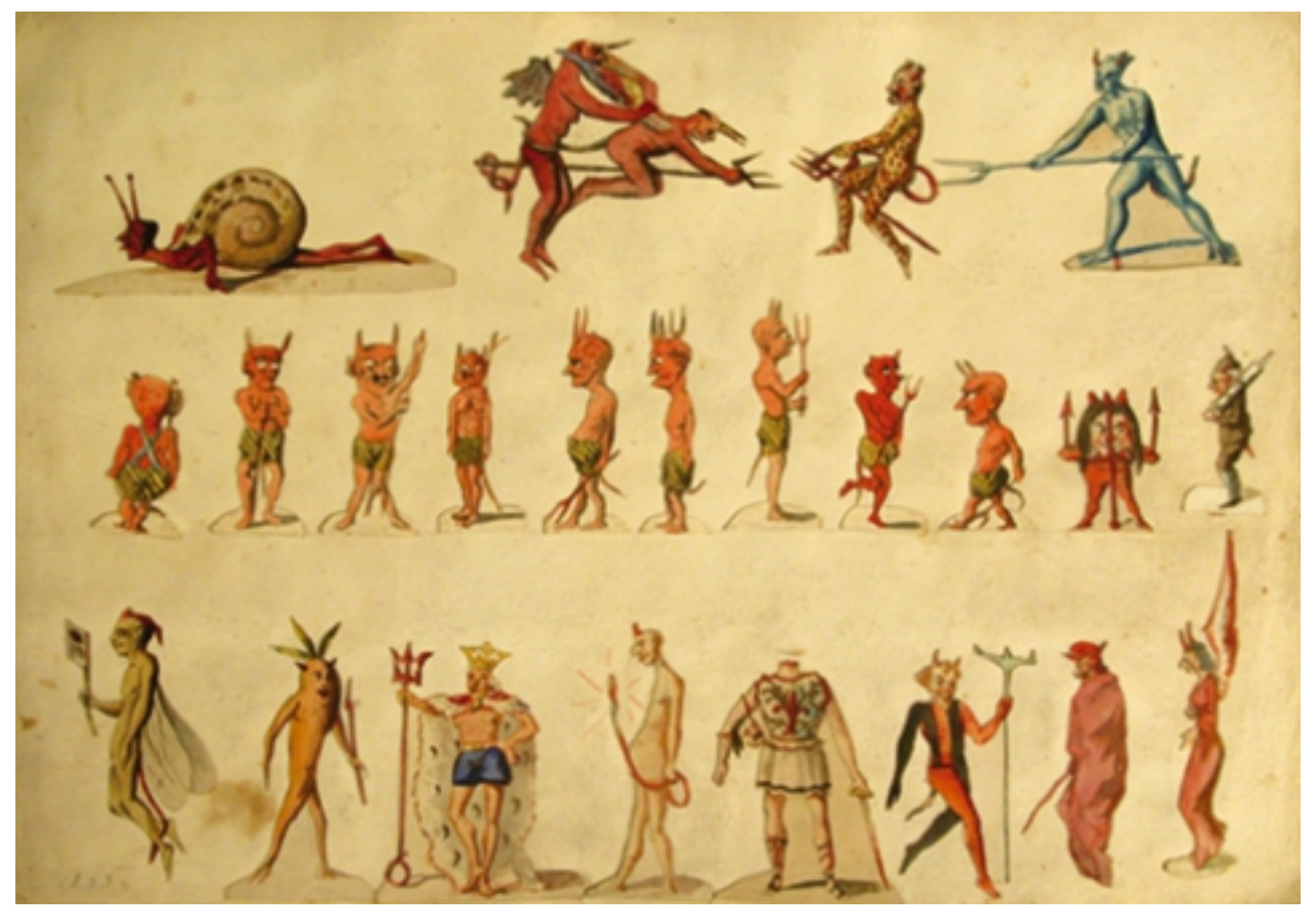

Figure 4 : Francesc Soler i Rovirosa (1836-1900). Maquette de costume pour La redoma encantada, de Juan Eugenio Hartzenbusch (1839), représentée au Teatre Principal de Barcelone (10/02/1874). Technique : mixte. MAE-Centre de Documentació i Museu de les Arts Escèniques. 


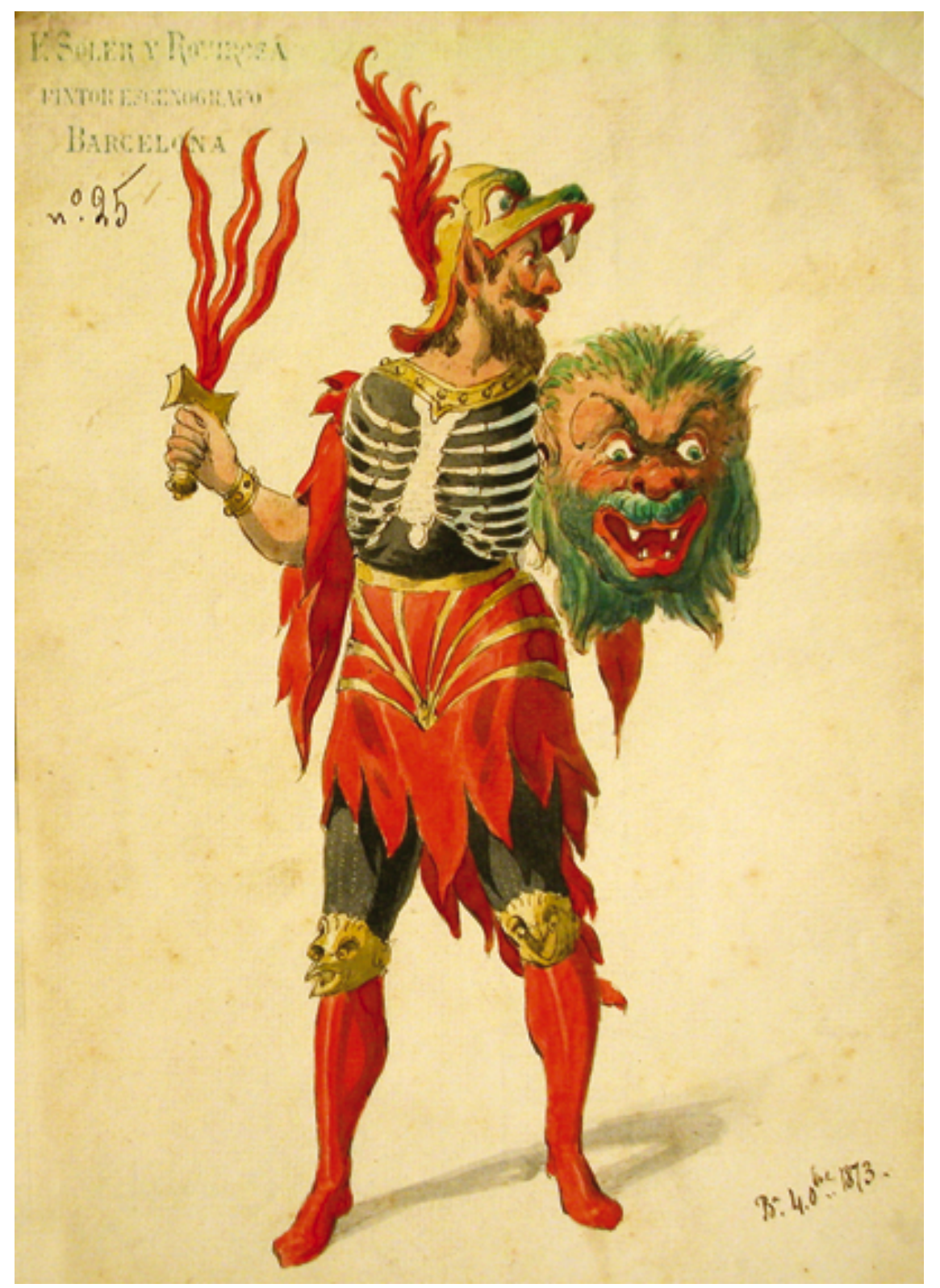

\section{Bibliographie}

La Comedia de Magia y de Santos, Congreso internacional Comedias de Magia y de Santos (siglos XVI-XIX), Madrid, Júcar, 1992.

Áldvarez Barrientos, Joaquín, 1992, "Apariencia y realidad en la comedia de magia dieciochesca », in La Comedia de Magia y de Santos, Congreso internacional Comedias de Magia y de Santos (siglos XVI-XIX), Madrid, Júcar, p. 341-349.

Aristote, 1980, La Poétique, Paris, Seuil.

Bettelneim, Bruno, 1976, Psychanalyse des contes de fées, Paris, Robert Laffont.

Bouissou, Sylvie, 2011, Crimes, cataclysmes et maléfices dans l'opéra baroque en France, Paris, Minerve.

Caro Baroja, Julio, 2010 [1961], Las brujas y su mundo, Madrid, Alianza Editorial.

CAVAILLÉ, Fabien, 2005, « Le malin plaisir : les perversions de la vengeance dans la tragédie baroque française », in Le théâtre et le mal, Revue d'études théâtrales, Presses Sorbonne Nouvelle.

Dubors, Claude-Gilbert, 2000, " La cruauté comme moteur dramatique et objet de représentation : un "baroquisme" théâtral (1452-1594) ", in Dumoulé, Camille (dir.), Les théâtres de la cruauté (Actes du colloque organisé à l'Université de Paris 10 - Nanterre 3-5 
décembre 1998), Paris, Desjonquères.

Fernández González, Manuel, 1852, La infanta Oriana, comedia de magia en cinco actos, en prosa y verso, Madrid, Vicente de Lalama, Biblioteca dramática.

Guerreau-Jalabert, Anita, 1994, « Fées et chevalerie », in Miracles, prodiges et merveilles au Moyen Âge, Société des historiens médiévistes de l'enseignement supérieur public, XXVe Congrès, Paris, Publications de la Sorbonne.

LAPLACE-Claverie, Hélène, 2007, Modernes féeries, le théâtre français du XXe siècle entre réenchantement et désenchantement, Paris, Champion.

Moliní́, Anne-Sophie, 2007, Corps ressuscitants et corps ressuscités, les images de la résurrection des corps en Italie centrale et septentrionale du milieu du XVe au début du XVIIe siècle, Paris, Honoré Champion.

\section{Notes}

1 Henri-René Lenormand, article de 1944 consacré au Grand Poucet, de Claude-André Puget, cité par Hélène Laplace-Claverie dans Modernes féeries, Paris, Champion, 2007, p. 106.

2 « Panteón subterráneo: al fondo una galería oscura; a la derecha del actor un sepulcro y otro a la izquierda; ambos en segundo término. Tras el de la derecha, un altar iluminado por una lámpara, y sobre él la estatua del ángel de la muerte. » (p. 17)

3 « Vestíbulo en el infierno; al fondo el trono de Satanás » (p. 20).

4 « Un genio horroroso » (p. 5).

5 « Varios diablos que traen a un hombre vestido de negro, con gorro y túnica de mágico, a quien los diablos figuran herir y desgarrar su cuerpo con garfios y tenazas » (p. 11).

6 « Genios infernales que improvisan un baile furioso » (p. 13).

7 « Ángel terrible y sombrío / que por mandato de Dios, / [es] guardián de las tumbas » (p. 17).

8 «- Oriana: Sombra que duermes aquí / bajo aqueste mármol frío, / despierta al acento mío / que desde el mundo te llama, / y alúmbrete de esta llama / el triste fulgor sombrío. » (La infanta deja delante del monumento la hojarasca que se enciende con una luz violenta y vivísima. La escena, que a medida que ha ido adelantando el monólogo de la infanta, ha ido oscureciendo hasta lo sumo, se ilumina con un relámpago; suena una detonación, y se abre el monumento de piedra, a través del cual aparece el rey Artus de Bretaña [...]. Desaparece el rey don Artus y se cierra el monumento) (p. 3-4).

9 « Hijo mío don Alvar, cuando leas este pergamino, la desgracia pesará sobre ti, y estarás encarcelado en la misma torre donde morirá tu padre. iVéngame! Un infame ha asesinado a tu madre, y yo siento los pasos del verdugo que se acerca. » (p. 17)

10 « Morirá, / [...] iMatarla!.. iTerrible idea! / iVerla gozando... reír / en los brazos de otro hombre!.. / iQue muera mil veces, sí! » (p. 19)

11 «Los monteros acomenten a los músicos. - Músicos: iFavor! iSocorro! iQue nos matan! - Gaiferos: iA ellos! iA ellos, hijos míos! - Alvar: iMalsines! Mi espada... iAy de mí! iConfesión! (riñen y cae herido don Alvar) - Sol: iDios mío! iMuerto! (cae desvanecida detrás de las almenas) » (p. 7).

12 « - Oriana: Dame ese licor / que a Fierabrás conquistaste [...] - Artus: ¿Quién va a morir? - Oriana: Un mancebo / a quien Merlín arrancó / padre y nombre [...] Alvar se llama. » (p. 3).

13 « iMuerto! sí, tieso, tiesísimo, quisiera llorar... quisiera... ello es que no sé... lo que yo quiero, lo que yo sé indudablemente... ya sé... lo que yo quiero es largarme de aquí más que a paso. » (p. 7).

14 La infanta Oriana, acte I, scène 16, " - Oriana: Por la virtud del bálsamo de Fierabrás que esta redoma contiene, torna a la vida, ensangrentado caballero (don Alvar empieza a volver en sí.) - Chorlito: iDiablo debéis de ser, que no infanta! Ved que ya mi señor abre los ojos. - Alvar: ¿En dónde estoy? ¿Qué ha pasado por mí? ¿Vivo aún? Sí, estoy vivo. (levantándose.) - Chorlito: No me atrevería yo a jurarlo. » (p. 7-8).

15 «La pieza de magia crea la ilusión "interna” de haber satisfecho las necesidades y 
resuelto los problemas de los personajes, pues no defrauda su empleo y castiga, de forma poco habitual en la realidad diaria, a los malos, para premiar a los buenos. »

16 «- Oriana: Tu hora ha llegado; la muerte orla tus ojos. Húndete. Ya se han abierto para ti las puertas de la eternidad... iTú al infierno!... iYo a mis alcázares! - Merlín: ¡Condenación! (desaparece por escotillón entre llamas)» (p. 20).

17 « El mismo mundo, desde el punto de vista moral, se divide en [...] dos partes claramente definidas y antagónicas [...]: la parte constituida por los cristianos que cultivan el Bien y las virtudes mismas y la formada por los que cultivan el Mal y los vicios. [...] Lo que antes [de la Cristianización] se había mantenido separado con arreglo a una división vertical, quedó separado con arreglo a una división horizontal. Ya no solamente había creencias rectas y creencias siniestras, torcidas: había creencias superiores y creencias inferiores ».

\section{Table des illustrations}

Figure 1 : Francesc Soler i Rovirosa (1836-1900). « Grand vestibule infernal ", maquette de décor pour La redoma encantada, de Juan

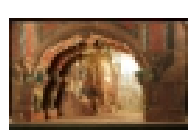

Titre Eugenio Hartzenbusch (1839), représentée au Teatre Principal de Barcelone (10/02/1874). Technique : bois et papier. MAE-Centre de Documentació i Museu de les Arts Escèniques.

URL http://america.revues.org/docannexe/image/961/img-1.jpg

Fichier image/jpeg, 252k

Figure 2 : Apel·les Mestres (1854-1936), maquette de costumes

Titre pour La almoneda del diablo, de Rafael María Liern (1864).

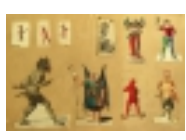
Technique : aquarelle. MAE-Centre de Documentació i Museu de les Arts Escèniques.

URL http://america.revues.org/docannexe/image/961/img-2.jpg

Fichier image/jpeg, 236k

Titre Figure 3 : Apel·les Mestres (1854-1936), maquette de costumes. MAE-Centre de Documentació i Museu de les Arts Escèniques.

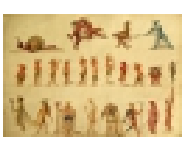

URL http://america.revues.org/docannexe/image/961/img-3.jpg

Fichier image/jpeg, 224k

Figure 4 : Francesc Soler i Rovirosa (1836-1900). Maquette de costume pour La redoma encantada, de Juan Eugenio

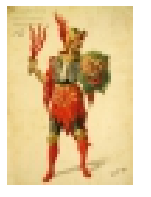

Titre Hartzenbusch (1839), représentée au Teatre Principal de Barcelone (10/02/1874). Technique : mixte. MAE-Centre de Documentació i Museu de les Arts Escèniques.

URL http://america.revues.org/docannexe/image/961/img-4.jpg

Fichier image/jpeg, 295k

\section{Pour citer cet article}

Référence papier

Lise Jankovic, « Le crime et le macabre sur scène dans La infanta Oriana (1852), comédie de magie de Manuel Fernández González », América, 43 | 2013, 79-90.

Référence électronique

Lise Jankovic, « Le crime et le macabre sur scène dans La infanta Oriana (1852), comédie de magie de Manuel Fernández González », América [En ligne], 43 | 2013, mis en ligne le 01 février 2015, consulté le 17 juin 2017. URL : http://america.revues.org/961; DOI :

10.4000/america.961

\section{Auteur}


Lise Jankovic

CREC, Université d'Artois

\section{Droits d'auteur}

Tous droits réservés 